Cómo citar este artículo: Quitian-Feliciano, I. D., Rodríguez-González, G. L., \& Morales-Rubiano, M. E. (2020). Desafíos de los centros de gestión de la investigación para promover la investigación colaborativa. Rev.investig.desarro.innov., 11 (1), 73-83.

\title{
Desafíos de los centros de gestión de la investigación para promover la investigación colaborativa
}

\section{Challenges of research management centers to promote collaborative research approach}

\author{
Iván Darío Quitian-Feliciano' \\ Gina Liseth Rodríguez-González ${ }^{2}$ \\ María Eugenia Morales-Rubiano ${ }^{3}$ \\ Recibido: diciembre 12 de 2019 \\ Aceptado: junio 05 de 2020
}

\begin{abstract}
Resumen
El objetivo es identificar los desafíos de los centros de investigación de la Universidad Militar Nueva Granada, para realizar investigación colaborativa. La Investigación tuvo un enfoque mixto, realizando 19 encuestas a líderes de los grupos de investigación y 8 entrevistas a directores de los centros de investigación. Para el $95 \%$ de los encuestados, fue importante que la Universidad haya promovido la investigación colaborativa, porque es enriquecedora para su actividad como investigadores; sin embargo, los requisitos en relación con el tipo de investigador externo con el que deben realizar la investigación colaborativa, son muy altos, estando el $83 \%$ de los encuestados en desacuerdo con ellos. Entre los desafíos a los que se enfrentan los directores de los centros de investigación, están: la necesidad de fomentar las redes científicas, la rigidez y el exceso de trámites administrativos internos, el perfil profesional de los investigadores y la falta de incentivos. Se concluye con la necesidad de flexibilizar los requisitos para hacer investigación colaborativa por parte de la universidad, de modo que se promueva una mayor participación de los investigadores en estas actividades.
\end{abstract}

Palabras clave: investigación colaborativa, innovación abierta, centros de investigación, generación de conocimiento.

\begin{abstract}
The objective is to identify the challenges of the research centers of the Universidad Militar Nueva Granada, to carry out collaborative research. The Research had a mixed focus. For this, 19 surveys were conducted to leaders of the research groups and 8 interviews with directors of the research centers. It was identified that for $95 \%$ of the respondents it was important that the University promoted collaborative research, because it is enriching for their activity as researchers. However, the University's goals and requirements in relation to the type of external researcher with whom they must carry out collaborative research are very high, with $83 \%$ of the respondents disagreeing. Among the most relevant findings are the challenges that the directors of the research centers face, such as the need to foster scientific networks, rigidity and excessive internal administrative procedures, the professional profile of researchers and the lack of incentives. It concludes with the need to make the requirements for collaborative research more flexible on the part of the university, so as to promote greater participation by researchers in these activities.
\end{abstract}

Keywords: collaborative research, open innovation, research centers, knowledge generation.

1 Administrador de Empresas, Universidad Militar Nueva Granada, Bogotá, Colombia. E-mail: u2204173@unimilitar.edu.co. ORCID: 0000-0002-4600-6219

2 Administradora de Empresas, Universidad Militar Nueva Granada, Bogotá, Colombia. E-mail: u2204142@unimilitar.edu.co. ORCID: 0000-0001-5050-2656

3 Administradora de Empresas, Magíster en Administración, Universidad Militar Nueva Granada, Bogotá, Colombia. E-mail: maria.morales@unimilitar.edu.co. ORCID: 0000-0002-5265-4466. 


\section{Introducción}

La investigación colaborativa, actualmente es un factor estratégico para la generación de conocimiento en las instituciones educativas. Gaviria, Mejía y Henao (2007), señalan que para tener una buena gestión del conocimiento en los centros de investigación, se deben propiciar espacios de encuentro entre los grupos de investigación. Esto debido a que la conversación es ante todo un encuentro entre pares, donde se demanda entrega y participación, se toman posiciones y es una manera de compartir conocimiento; sin embargo, este no ha sido un proceso fácil para los actores involucrados porque existen barreras $y$ desafíos por superar.

El propósito de este documento, es identificar los desafíos que tienen los directores de centros de investigación de la Universidad Militar Nueva Granada (UMNG), para realizar investigación colaborativa, derivado de la decisión de la Universidad de promover este tipo de investigación, como nuevo criterio de evaluación para las convocatorias de financiación de proyectos de investigación (UMNG, 2019).

La calidad en la gestión del conocimiento en los centros de investigación, es algo que preocupa a las instituciones educativas tanto privadas como públicas, más si se piensa en dar el paso de promover la investigación colaborativa (Rodríguez, 2016). Con esta investigación se espera conocer el impacto y los retos que trajo la decisión de trabajo colaborativo, tanto para los investigadores como para los líderes de grupos y directores de centros de investigación de la UMNG. Así será posible generar una contribución en la comprensión del proceso y las implicaciones de un enfoque de investigación colaborativa, que permita adelantar acciones desde la gestión de las diferentes unidades de apoyo que tiene el investigador.

En la siguiente sección se presentan los conceptos y el rol que juegan los centros de investigación en la actualidad, para desarrollar investigación colaborativa o innovación abierta. Seguidamente, se hace referencia a los enfoques de colaboración y los actores involucrados. Luego, se ilustra la metodología que se aplicó en el desarrollo de la investigación. Después, se presentan el análisis y discusión de los resultados. Por último, se encuentran las conclusiones derivadas de la investigación.

\section{Marco teórico y metodología}

\subsection{Centros de investigación}

En el contexto actual, el conocimiento creado en las universidades se ha visto influenciado por tener una visión abierta al desarrollarlo. Autores como Gros y Lara (2009), afirman que hay una mayor globalización de la investigación y desarrollo, donde la tecnología ha facilitado los procesos de comunicación, de acceso a la información y de creación del propio conocimiento, lo que ha exigido que ahora las instituciones se relacionen con la industria, la sociedad, centros tecnológicos y de investigación, entre otros, con el fin de crear conocimiento que pueda aportar de manera objetiva al crecimiento económico.

Los centros de investigación, según Colciencias (2019), "son organizaciones públicas, privadas o mixtas dedicadas a la generación de conocimiento fundamental para el país, mediante proyectos de investigación científica básica y/o aplicada en líneas de investigación específicas". Es decir, el conocimiento creado debe aportar a la solución de aspectos fundamentales en los que el país necesite desarrollo, lo que implica que los centros de investigación se relacionen con actores externos para generar nuevo conocimiento. En este sentido, como lo afirman Berrío, Angulo y Gil (2013), las investigaciones más que tener gestión del conocimiento, implementen una cultura innovadora tomando el conocimiento no solo como un simple proceso más en la institución, sino preocupándose por generar valor a ese conocimiento, para así afrontar las problemáticas actuales que viva la sociedad y los mismos centros de investigación en su papel como desarrolladores de conocimiento. 
Los centros de investigación universitarios, son unidades creadas para apoyar los procesos de investigación e innovación de la comunidad académica $y$, junto con otras estructuras, sirven de enlace con el entorno. La apertura de los centros de investigación también implica la innovación abierta, porque permite que la universidad genere participación de agentes externos para la captación de nuevas ideas y recursos, lo cual potencializa a la universidad al hacerla innovadora y en algunos casos, es la clave del éxito en los desarrollos educativos, porque hace que se creen nuevos conocimientos y que se originen procesos creativos (Sánchez, Pedrosa \& Maldonado, 2015).

\subsection{Investigación colaborativa}

Los avances en innovación abierta aún son escasos y más cuando se relaciona con investigación universitaria; sin embargo, la investigación colaborativa ha sido considerada como una forma de desarrollar este tipo de innovación en el ambiente educativo. Chen, Zhang y Fu (2017), consideran que es imposible que hoy un país se mantenga alejado de las colaboraciones en investigación con otros países, debido a que la ciencia, la tecnología y la innovación cada vez más se convierten en una empresa global y colaborativa. Por lo tanto, se hace necesario que los países generen políticas que den apertura a las investigaciones colaborativas, entre los actores del sistema nacional de ciencia, tecnología e innovación, pero donde estas relaciones también se establezcan internacionalmente.

Se destacan países industrializados como Estados Unidos, y en América Latina, Brasil, porque han trabajado este tema entre el sector empresarial y el académico para generar la investigación de co-creación e innovación abierta (Ramírez \& García, 2017), lo que muestra que estas aperturas han traído ventajas competitivas para los países. Chen et al. (2017), sugieren que en el campo de la investigación colaborativa internacional, los responsables políticos deben tomar medidas efectivas para intensificar los vínculos de colaboración con otros países. No obstante, añaden que no todos los países tendrán el mismo beneficio, pues aquellos que están en desarrollo tendrán una oportunidad aún más grande, debido a que podrán aprovechar los recursos de investigación y desarrollo con los que cuentan los países desarrollados. También consideran que es probable que los países industrializados restrinjan la colaboración con países en desarrollo, porque su competitividad global en innovación es mucho mayor.

\subsection{Enfoques de colaboración y autores involucrados}

\section{Relación industria-universidad}

La relación industria-universidad es una de las de mayor impacto, por el conocimiento innovador y las ideas que se han generado en el desarrollo de investigaciones. Lee y Win (2004), consideran que la transferencia ideal se da en el trabajo conjunto entre centros de investigación y la industria, porque complementa cada organización, instalaciones y experiencia, generando mejoras en las capacidades de las industrias locales a través de programas de investigación, donde es posible compartir y reducir el riesgo. Por esto, existe un gran interés de los investigadores universitarios a nivel individual, de crear conocimiento a partir de una mayor colaboración (Boardmana \& Corley, 2008).

Las empresas también han sentido la necesidad de ser más abiertas, y para generar innovaciones, han buscado acceso a un conjunto más amplio y complejo de conocimiento técnico-científico de lo que antes era necesario, adoptando prácticas de innovación abierta, para facilitar el desarrollo de estrategias efectivas de competencia (Petroni, Venturini \& Verbano, 2012). En este sentido, se empiezan a establecer relaciones con los centros de investigación para hacer investigación colaborativa, de modo que sus procesos de innovación sean más eficientes. Esto exige que las organizaciones modifiquen sus estructuras y el perfil del personal, para lograr mejores relaciones al 
momento de establecer un enfoque de innovación abierta.

Esta relación se ha visto afectada en varios casos por el factor financiero. Lee y Win (2004), y Boardmana y Corley (2008), concuerdan en que para que la colaboración tenga éxito, es necesario que ambas partes, tanto centros de investigación como industria, compartan costos e instalaciones, porque en muchas ocasiones los centros de investigación se dedican a ofrecer servicios de bajo riesgo, donde en caso de no obtener los resultados esperados, la organización como fuente única de financiamiento, es la que más pierde al realizar ese tipo de inversiones. Adicionalmente, Muriithi, Horner, Pemberton y Wao (2017), identificaron que más allá del gasto financiero que debe hacerse en una investigación colaborativa, lo que dificulta una buena relación con la industria, de acuerdo con la percepción de los investigadores, es que la industria no confía en lo que hacen los investigadores, limitando la motivación para ambas partes en llevar a cabo investigación colaborativa.

En este contexto también se ha considerado importante el tamaño de la organización, pues el enfoque de cada una, afectará el porqué crear una relación con los centros de investigación. McKelvey, Zaring y Ljungberg (2014), afirman que las diferencias radican en que la empresa pequeña ve a la universidad como una herramienta "solucionadora de problemas", en temas especialmente relacionados con el mercado y no en temas tecnológicos donde la visión de los centros es a largo plazo con proyectos extensos. Al contrario, la empresa grande, al tener la posibilidad de manejar la cartera del proyecto, busca que la colaboración se base de acuerdo con su tecnología central, captando conocimiento a través de la relación con personas altamente capacitadas de los centros de investigación. En este punto, es necesario que los directores de los centros al realizar su gestión, tengan en cuenta este factor para saber qué interacciones deben promover con la industria, y cómo lograr que los investigadores e interesados se relacionen de la forma que se desea (Boardman \& Corley, 2008).

\section{Relación universidad-comunidad}

Las universidades y las comunidades aprenden las unas de las otras cuando realizan un proceso de colaboración, impulsando nuevas maneras de trabajar juntas, de crear conocimientos y de afrontar problemáticas sociales complejas. Un aspecto fundamental de esta colaboración, se relaciona con las estructuras de búsqueda de colaboración entre las comunidades y la universidad, ya que estas deben: estimular, dinamizar e impulsar la colaboración investigativa, con compromiso comunitario entre académicos y expertos de las comunidades. Cuando los investigadores universitarios trabajan en colaboración con grupos y organizaciones comunitarias, influyen en un cambio de mentalidad colectivo a través del intercambio de conocimiento. Es decir, se trata de un proceso que permite crear nuevos conocimientos, mediante: la generación, la movilización y el uso del conocimiento de una manera colaborativa (Lall, 2011).

Para entender la forma y función de los centros de investigación, Lall (2011), destaca que las estructuras de investigación colaborativa de apoyo, pueden tomar diferentes formas e incidir en el cambio social y sistémico a través de su impacto en las políticas y las prácticas, contribuyendo así al interés general en ámbitos locales, regionales, nacionales e internacionales. Esto permite: ampliar el proceso de participación, afrontar las desigualdades sociales y económicas, resolver los problemas financieros, políticos y éticos, la innovación y el diseño en la docencia, la investigación y el plan de estudios, gestionar las relaciones de trabajo entre la comunidad y las redes de aprendizaje que constituyen el núcleo de la universidad.

\section{Relación entre centros de investigación}

Las investigaciones adelantadas bajo un enfoque colaborativo, han tomado valor para los investigadores al crear vínculos entre disciplinas o 
interdisciplinarios, y con centros de investigación tanto internos como de universidades externas, porque es una forma de resolver problemas complejos; sin embargo, se han encontrado diversos factores que influyen en el desarrollo de este tipo de investigaciones. Rijnsoever y Hessels (2010), identificaron que en las investigaciones entre disciplinas: los años de experiencia laboral, haber trabajado anteriormente en otras universidades $\mathrm{u}$ otros institutos de investigación, el rango académico y las dinámicas del campo científico, se relacionan positivamente con la colaboración; además, en la investigación colaborativa interdisciplinaria, no influye la formación académica, pero el género y trabajar en una disciplina estratégica, se relaciona positivamente en la colaboración.

Muriithi et al. (2017), afirman que algunos de los factores que influyen en la conducta de los investigadores y en el éxito de las colaboraciones en investigación, son de naturaleza personal, como: el nivel de habilidades y experiencia, el nivel de compromiso, los estilos de trabajo individuales y las cuestiones éticas. Adicionalmente, hay factores ambientales externos en oposición a los factores personales, en los que un investigador individual no tiene control directo, como la disponibilidad de recursos y los factores institucionales, que afectan el desarrollo de una investigación colaborativa. Lauto y Sengoku (2014), consideran que la gestión que exista dentro de los centros de investigación, influye al momento de realizar investigación colaborativa, debido a que son los que refuerzan e incentivan a los científicos, para hacer investigación interdisciplinaria a través de una cultura que la promueva, aun cuando la percepción del investigador y su rango académico (postdoctorados), puede afectar su interés por llevar a cabo investigación colaborativa.

Diversos autores coinciden en que los incentivos que promuevan los centros y la gestión con respecto a: recursos, el liderazgo, su capacidad de coordinación y dirección y los vínculos creados con las demás instituciones, son aspectos necesarios para llevar a cabo una investigación colaborativa (Boardmana \& Corley, 2008; Muriithia et al., 2017; Rijnsoever \& Hessels, 2010). Esto con el fin de que sea una colaboración exitosa, donde el investigador se motive a crear conocimientos con otras instituciones o investigadores. Asimismo, Zuo y Zhao (2018), afirman que aquellas instituciones que son multidisciplinarias, tienen más probabilidad de fomentar la interdisciplinariedad en la investigación, pero este no debe ser impedimento para que otras generen colaboraciones. Estos autores sugieren para aquellas instituciones multidisciplinarias, que enfoquen los esfuerzos en la coordinación, administración e incentivos para explotar completamente los beneficios potenciales de la diversidad multidisciplinaria, y así estimular más colaboraciones de investigación dentro de la organización.

\subsection{Metodología}

La investigación tuvo un enfoque cuantitativo y cualitativo. Primero, se enviaron 40 encuestas a los líderes de los grupos de investigación por medio electrónico, de las cuales se obtuvieron 19 respuestas, que buscaban identificar: sus experiencias a la hora de realizar investigación colaborativa, las características personales de un investigador para llevar a cabo este tipo de investigación, los factores externos que pueden influir y las dificultades del proceso para realizar una investigación colaborativa con otra institución. Luego, se procedió a realizar una entrevista semi-estructurada a los 8 directores de los centros de investigación de la UMNG, pertenecientes a las facultades de: Ciencias Económicas, Ciencias Básicas, Derecho, Humanidades, Ingeniería, Medicina, Relaciones Internacionales y Estudios a Distancia, para indagar sobre las opiniones que tenían sobre el tema de investigación desde su posición de directores.

Se realizó una revisión de literatura, que llevó a la identificación de cuatro categorías que permiten indagar sobre los retos que tienen los centros de investigación de la UMNG, para realizar investigación colaborativa, a saber: las características 
personales que debe tener un investigador para realizar investigación colaborativa; las barreras para realizar investigación colaborativa con la comunidad académica interna y externa; el impacto de promover investigación colaborativa como nuevo criterio de evaluación en los proyectos de investigación; y los desafíos de los directores de centros y líderes de grupos de investigación de la UMNG para hacer investigación colaborativa. Alrededor de estas categorías, se construyeron las preguntas de las encuestas y las entrevistas.

Para el análisis de la información, se realizó la transcripción de las entrevistas para poder identificar las categorías más relevantes, previamente establecidas, obtener información más detallada de subcategorías y de esta forma tomar los aspectos clave, de acuerdo con las repeticiones o apariciones. En cuanto a las encuestas, se procedió a descargar la información de los formularios electrónicos y consolidarla en un Excel para tabular los datos.

\section{Resultados y discusión}

3.1 Características del investigador para realizar investigación colaborativa

Los líderes de los grupos de investigación de la Universidad, están en un 95\% de acuerdo en que las características personales determinantes de los investigadores al aplicar la investigación colaborativa, están dadas en relación con su nivel de: habilidades, experiencia, estilo de trabajo grupal e individual y cuestiones éticas. En las entrevistas, los directores de centros de investigación destacan que es importante tener una comunicación asertiva y empatía, basar la relación en el respeto, responsabilidad y compromiso, siempre actuando de manera ética. Afirman que el "querer" hacer investigación colaborativa por parte del investigador, es necesario para poder obtener los resultados esperados y que la investigación funcione. También los directores agregan que el bilingüismo, ser flexible a nuevas ideas, la experiencia investigativa y el conocer sobre lo que se quiere investigar, serán características fundamentales para llevar a cabo una investigación colaborativa.

Lo anterior permite deducir que tanto los líderes de grupos de investigación como los directores de centro, concuerdan en varias características; sin embargo, los directores resaltan el "querer" o voluntad del investigador, como factor que facilita que las investigaciones colaborativas se lleven a cabo, debido a que la sola obligación de cumplir un requisito, no garantiza el éxito de las relaciones investigativas.

\subsection{Barreras para realizar investigación colaborativa}

Al realizar el análisis de las entrevistas y encuestas, se encontró que los líderes de grupos y los directores de centros identifican ciertas limitaciones al desarrollar sus investigaciones, que han obstaculizado con el paso del tiempo poder efectuar investigación colaborativa. El $61 \%$ de los líderes, considera que los trámites administrativos de la Universidad son excesivos, burocráticos, desgastantes, que no se ajustan a las necesidades del investigador y que, al momento de tener relación con un ente externo, la investigación se vuelve dispendiosa. Además, la no paridad entre las condiciones o requisitos exigidos en la UMNG con respecto a otras universidades, genera un desequilibrio e impide que se puedan establecer relaciones con otros investigadores, para llevar a cabo una investigación colaborativa.

Además, para el $95 \%$ de los encuestados, fue muy importante que la UMNG haya promovido la investigación colaborativa, porque es un acto enriquecedor para su desarrollo como investigadores; sin embargo, las metas y requisitos de la Universidad, con relación al tipo de investigador externo con el que debe realizar investigación colaborativa, son muy altos, mostrándose el $83 \%$ de los encuestados en desacuerdo, porque son requisitos que muchas veces ni los mismos investigadores de la UMNG cumplen. En la tabla 1, se muestran las barreras que tienen los directores de los centros de investigación de la Universidad, que limita la realización de investigaciones colaborativas. 
Tabla 1. Barreras para realizar investigación colaborativa interna y externa.

\begin{tabular}{ll}
\hline No. & Barreras \\
\hline 1 & Falta de redes científicas del investigador \\
2 & Sometimiento de la institución externa a los requerimientos de la UMNG \\
3 & Exceso de trámites administrativos internos \\
4 & Poco tiempo \\
5 & Falta de motivación \\
6 & Perfil profesional del investigador \\
7 & Metas muy altas \\
8 & Se ve como un requisito, no como un desafío \\
9 & Poco interés de la comunidad académica externa \\
\hline
\end{tabular}

Los directores de centro agregan que la Universidad, ni de manera interna ni externa, genera espacios para que los investigadores amplíen sus redes científicas, lo cual limita y genera temor en el investigador para realizar este tipo de investigación. Además, concuerdan con los líderes de grupo, en que los procesos administrativos de la Universidad son desgastantes y toman mucho tiempo, haciendo que el investigador externo se desanime en la realización de la investigación colaborativa.

3.3 Impacto de promover investigación colaborativa como criterio de evaluación

Los directores de centro consideran que la implementación de la investigación colaborativa, ha creado la necesidad en los investigadores de buscar un par externo y de cambiar su manera de desarrollar investigación, puesto que por mucho tiempo (hasta las convocatorias de investigación de la UMNG 2018), no se había agregado como criterio de evaluación; sin embargo, plantean que este cambio radical en las convocatorias, sin un proceso de socialización y con niveles altos, generó malestar en los investigadores.
Asimismo, los directores indican que hubo una disminución de los proyectos de investigación en la última convocatoria. Ahora bien, los que se postularon, mejoraron su calidad notoriamente, presentándose en cinco de ocho centros de investigación, casos exitosos de investigación colaborativa. Esto hace que la Universidad cada vez esté mejor posicionada en relación con la investigación, y que se promueva el interés por parte de externos para investigar con la UMNG.

3.4 Desafíos para directores de centro y líderes de grupos de investigación

En la figura 1, se presentan los principales desafíos identificados en las encuestas por líderes de los grupos de investigación, para realizar investigación colaborativa. Ellos indican la necesidad de que la dirección de la Universidad cambie la manera en que desarrolla sus procesos, teniendo en cuenta las perspectivas y necesidades de los investigadores; además, recomiendan modificar los procesos administrativos para llevar a cabo una investigación colaborativa y, especialmente, crear espacios para generar redes científicas. 


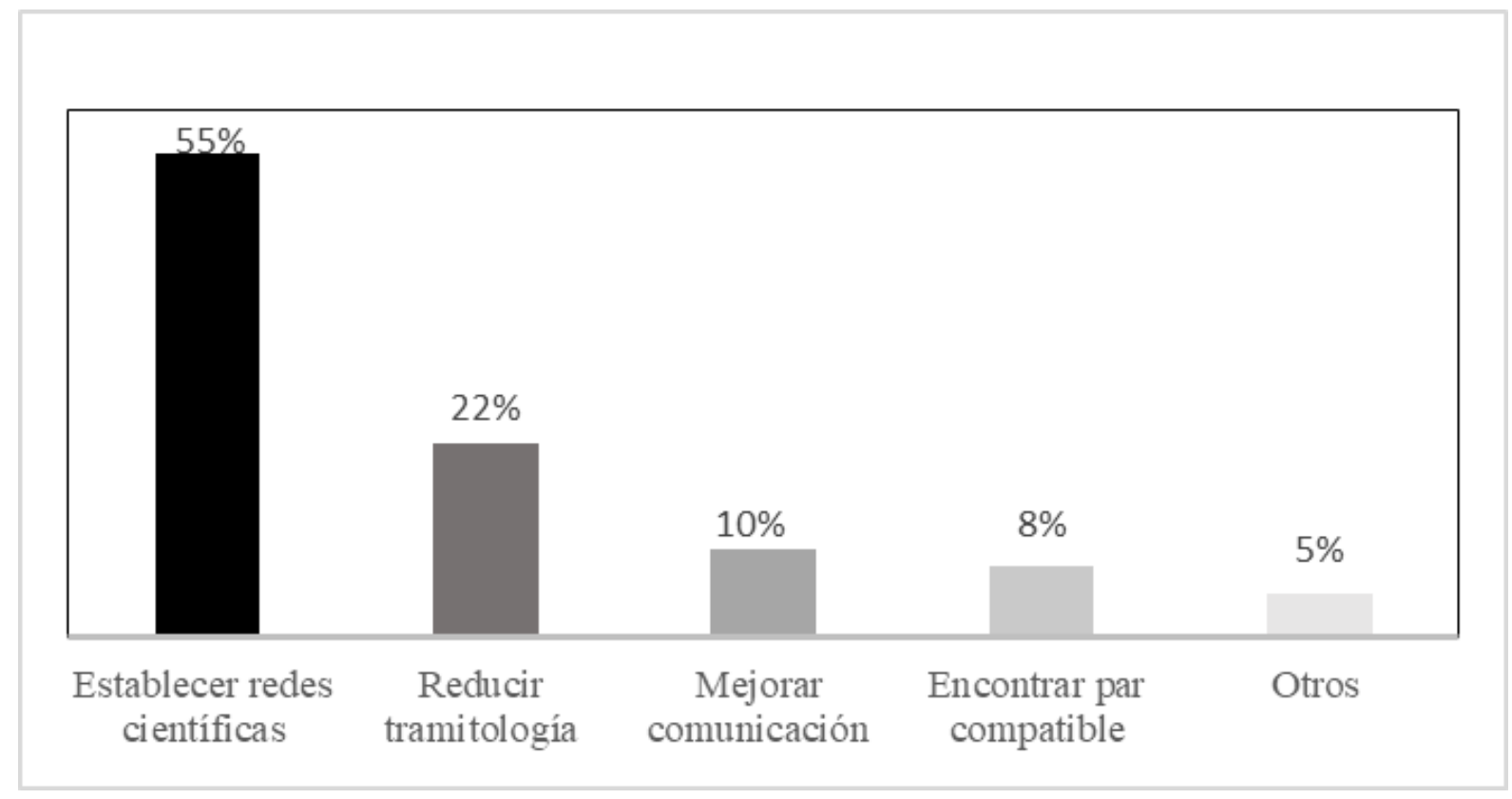

Figura 1. Desafíos para realizar investigación colaborativa.

Por su parte, los directores de centro consideran que, desde la Vicerrectoría de Investigaciones de la Universidad, se deben plantear estrategias y políticas nuevas, para poder generar más proyectos de investigación colaborativa, que faciliten la creación de redes y motiven a los investigadores. En este sentido, consideran importante la generación de espacios, en los que se pueda conocer a otros investigadores de las distintas facultades de la UMNG, para que desde estos espacios puedan formularse y construirse nuevos proyectos de investigación colaborativa.

Asimismo, agregan que es necesario que los directores de centro cambien su papel. Es decir, que su labor no sea netamente administrativa, sino que sean un medio para promover y apoyar la investigación colaborativa, que sean quienes, a través de la asistencia a eventos de investigación, creen las redes científicas que el centro necesita, nutriéndose de nuevas ideas. Otro de los desafíos, es que la universidad debe definir cuál es su fortaleza en investigación, ya que esto hará que cuando se establezca una investigación colaborativa, bien sea con investigadores, industria o comunidad, de confianza al externo, se tenga claro cuál será el enfoque y en qué se podrán ofrecer soluciones.

\subsection{Discusión}

Cabe destacar que, de acuerdo con la revisión literaria realizada y la obtención de información primaria a partir de las encuestas y entrevistas llevadas a cabo a líderes de grupos y directores de los centros de investigación, se encontraron diversos contrastes entre lo teórico y lo práctico, lo que a continuación se presenta.

Boardmana y Corley (2008), afirmaron que los investigadores cada vez están más motivados en realizar investigación colaborativa, porque les permite ampliar sus conocimientos. Los investigadores de la UMNG concuerdan en que el aplicar este tipo de investigación es enriquecedor para ellos, lo que se evidencia en que el $95 \%$ estuvo de acuerdo con el criterio de evaluación en las convocatorias, para financiación de los nuevos proyectos de investigación, pues se fortalece la investigación en la Universidad. 
Muriithi et al. (2017), definieron algunas de las características personales que debe tener un investigador al realizar investigación colaborativa, como: el nivel de habilidades y experiencia, el nivel de compromiso, los estilos de trabajo individuales y las cuestiones éticas, las cuales fueron contrastadas dentro de las encuestas realizadas, donde el 94,5\% de los encuestados estuvieron de acuerdo; sin embargo, los directores de centros manifestaron que el "querer" realizar este tipo de investigación, debe estar en el investigador y debe ser un aspecto a tener en cuenta en su desarrollo. Esto se relaciona estrechamente con lo afirmado por Domínguez, Crhová y Molina (2015), quienes consideran: la motivación, las creencias, el interés y la actitud del investigador como factores importantes para el desarrollo de investigaciones colaborativas.

Los líderes de grupos y directores de investigación de la Universidad, afirmaron que los procesos administrativos para llevar a cabo investigación colaborativa, especialmente con externos, era un proceso desgastante y desalentador. Muriithi et al. (2017), encontraron que dentro de los factores externos que puede afectar el desarrollo de una investigación, están los procesos institucionales de las partes, por lo que es importante tener en cuenta este aspecto como un desafío para la Universidad.

En cuanto a los desafíos, se plantea que los directores de los centros de la UMNG deben cambiar su papel meramente administrativo a uno más dinámico, que dé apertura a las investigaciones colaborativas. Este aspecto se relaciona con lo afirmado por Boardman y Corley (2008), quienes señalan que es necesario que el director del centro gestione, de tal manera que logre encontrar aquellas relaciones que favorecen su interacción con la industria u otro ente externo. Además, Lauto y Sengoku (2014), consideran que quienes gestionan los centros de investigación, deben ser quienes motiven e incentiven que los científicos realicen investigación colaborativa.
Rijnsoever y Hessels (2010), indicaron que los aspectos profesionales son importantes en el desarrollo de una investigación. Esto coincide con lo afirmado por los directores de centro, pues ellos consideran que el nivel de formación y la experiencia en investigación, juegan un papel importante en el desarrollo de la investigación colaborativa. Otro aspecto importante en la realización de investigación colaborativa, es la relación de una institución con las demás (Boardmana \& Corley, 2008; Muriithia et al., 2017; Rijnsoever \& Hessels, 2010). En este caso, se observa que mientras para los directores de los centros es necesario que los científicos creen sus propias redes científicas, estos plantean que debe ser también la Universidad quien fomente las buenas relaciones con las demás.

En el marco conceptual de este trabajo, se observa que los autores reconocen que los recursos y el financiamiento dentro de la investigación colaborativa, es un factor importante que la dificulta; sin embargo, en los resultados de la investigación, ninguno de los líderes o directores planteó este tema como impedimento para llevar a cabo este tipo de investigación. Otro de los hallazgos es que, dentro de la UMNG, aquellas facultades que tienen solo postgrados han obtenido mejores resultados en la ejecución de proyectos colaborativos, debido a que el nivel de formación de los estudiantes les ha permitido crear redes, que les facilitan el encontrar con quien investigar.

\section{Conclusiones}

Se puede concluir que los procesos administrativos de la UMNG, al momento de llevar a cabo una investigación colaborativa, deben ser más flexibles, eliminando el exceso de trámites que generan tiempos adicionales y un desgaste o desánimo por parte de los investigadores, lo que facilitaría crear relaciones con los pares. Este punto también es importante para la comunidad educativa externa, debido a que universidades o instituciones que deseen realizar investigación colaborativa con la UMNG, al ver que se enfrentan 
a trámites excesivos que ponen en riesgo el cronograma y los tiempos pactados para la investigación, prefieren no realizar la investigación o los que empiezan, la abandonan poniendo en riesgo el éxito del trabajo conjunto.

Los hallazgos también permitieron identificar las principales características que debe tener el investigador al hacer investigación colaborativa, tales como: comunicación asertiva, ética, experiencia en investigación, responsabilidad y voluntad, entre otras.

En cuanto a la gestión de los directores de los centros de investigación, esta debe tener un equilibrio entre lo administrativo, la gestión y lo investigativo, tener un liderazgo donde se promuevan espacios para incentivar la investigación colaborativa en sus investigadores. Asimismo, deberían ser ellos quienes creen y participen de eventos de investigación, donde se puedan ampliar las redes científicas. Igualmente, los directores deberían realizar un acompañamiento más cercano a la investigación colaborativa, para que el investigador sienta el apoyo y motivación durante el proceso.

Finalmente, se concluye que los criterios de evaluación planteados por la Universidad para promover la investigación colaborativa con un par externo, son muy altos para el tipo de investigador que posee, especialmente en los centros de investigación en los que se trabaja con pregrados. En este sentido, se considera importante ajustar estos criterios, de tal manera que en vez de limitar las investigaciones que promueven los investigadores, den apertura a estas para que la UMNG adquiera experiencia en este tipo de investigaciones $y$, con el tiempo, se puedan ir modificando estos requisitos.

\section{Agradecimientos}

Artículo derivado del Proyecto de Iniciación Científica PIC-ECO-2864: Desafíos para la gestión de centros de investigación de la UMNG en un enfoque de investigación colaborativa o de innovación abierta, vigencia 2019, financiado por Vicerrectoría de Investigaciones de la Universidad Militar Nueva Granada.

\section{Referencias}

Berrío, H., Angulo, F., \& Gil, I. (2013). Gestión del conocimiento como bases para la gerencia de centros de investigación en universidades públicas. Revista Dimensión Empresarial, 11 (1), 116-125.

Boardmana, C., \& Corley, E. (2008). University research centers and the composition of research collaborations. Revista Research Policy, 37, 900-913.

Chen, K., Zhang, Y., \& Fu, X. (2017). International research collaboration: ¿An emerging domain of innovation studies? Revista Research Policy 48, 149-168. doi: https://doi.org/10.1016/j. respol.2018.08.005

Colciencias. (2019). Centros / Institutos de Investigación. Recuperado de: https://www.colciencias. gov.co/portafolio/reconocimiento_de_actores/ centros-institutos-investigacion

Domínguez, M. Crhová, J., \& Molina, R. (2015). Investigación colaborativa: Las creencias de los docentes de lenguas. Revista Iberoamericana de Educación Superior, 6 (17), 119-134. Recuperado de: https://www.sciencedirect.com/science/ article/pii/S2007287215000396

Gaviria, M. Mejía, M., \& Henao, M. (2007). Gestión del conocimiento en los grupos de investigación de excelencia de la Universidad de Antioquia. Revista Interamericana de Bibliotecología, 30 (2), 137-163.

Gros, B., \& Lara, P. (2009). Estrategias de innovación en la educación superior: El caso de Universitat Oberta de Catalunya. Revista iberoamericana de educación, 49, 223-245. 
Lall, N. (2011). Estructuras de investigación colaborativa comunidad-universidad: aproximación a su posible impacto. Rizoma freireano, 9. Recuperado de: http://www.rizoma-freireano.org/ estructuras-de-investigacion-colaborativa-comunidad-universidad-aproximacion-a-su-posible-impacto--nirmala-lall

Lauto, G., \& Sengoku, S. (2014). Perceived incentives to transdisciplinarity in a Japanese university research center. Revista Futures 65, 136-149.

Lee, J., \& Win, H. (2004). Technology transfer between university research centers and industry in Singapore. Revista Technovation, 24, 433-442.

McKelvey, M., Zaring, O., \& Ljungberg, D. (2014). Creating innovative opportunities through research collaboration: An evolutionary framework and empirical illustration in engineering. Revista Technovation, 39-40, 26-36. doi: http://dx.doi. org/10.1016/j.technovation.2014.05.008

Muriithi, P., Horner, D., Pemberton, L., \& Wao, $\mathrm{H}$. (2017). Factors influencing research collaborations in Kenyan universities. Revista Research Policy, 47, 88-97. doi: http:// dx.doi.org/10.1016/j.respol.2017.10.002

Petroni, G., Venturini, K., \& Verbano, C. (2012). Open innovation and new issues in $R \& D$ organization and personnel management. The International
Journal of Human Resource Management, 23 (1), 147-173. doi: 10.1080/09585192.2011.561250

Ramírez, M., \& García, F. (2017). Co-creation and open innovation: Systematic literature review. Revista Comunicar, 54 (26), 09-18. doi: https://doi. org/10.3916/C54-2018-01

Rijnsoever, F., \& Hessels, L. (2010). Factors associated with disciplinary and interdisciplinary research collaboration. Revista Research Policy, 40, 463-472. doi: 10.1016/j.respol.2010.11.001

Rodríguez, M. (2016). La gestión del conocimiento en los centros de investigación. Revista Entramados-Educación y Sociedad, 3, 123-132. Recuperado de: https://fh.mdp.edu.ar/revistas/index.php/ entramados/article/view/1624/0

Sánchez, P., Pedrosa, C., \& Maldonado, C. (2015). Innovación abierta en entornos educativos. Revista Opción, 31(4), 602-616. Recuperado de: https:// www.redalyc.org/pdf/310/31045569036.pdf

UMNG. (2019). Investigación. Convocatorias Internas VICEIN. Recuperado de: http://www. umng.edu.co/convocatorias

Zuo, Z., \&Zhao, K. (2018).The more multidisciplinary the better? - The prevalence and interdisciplinarity of research collaborations in multidisciplinary institutions. Journal of Informetrics, 12, 736-756. doi: https://doi.org/10.1016/j.joi.2018.06.006 
